\title{
Transient cold storage prior to normothermic liver perfusion may facilitate adoption of
}

\section{a novel technology}

Carlo DL Ceresa $^{1}$, David Nasralla ${ }^{1}$, Christopher JE Watson ${ }^{2}$, Andrew J Butler ${ }^{2}$, Constantin C Coussios $^{3}$, Keziah Crick ${ }^{2}$, Leanne Hodson ${ }^{4}$, Charles Imber ${ }^{5}$, Wayel Jassem ${ }^{6}$, Simon R Knight ${ }^{1}$, Hynek Mergental $^{7}$, Rutger J Ploeg${ }^{1}$, Joerg M Pollok ${ }^{5}$, Alberto Quaglia ${ }^{6}$, AM James Shapiro ${ }^{8}$ Annemarie Weissenbacher ${ }^{1}$, Peter J Friend ${ }^{1}$

1. Nuffield Department of Surgical Sciences, University of Oxford, Oxford, UK

2. University of Cambridge Department of Surgery, Addenbrooke’s Hospital, Cambridge, UK

3. Institute of Biomedical Engineering, Department of Engineering Science, University of Oxford, Oxford, UK

4. Oxford Centre for Diabetes, Endocrinology and Metabolism, University of Oxford, Oxford, UK

5. Department of Hepatopancreatobiliary and Liver Transplant Surgery, Royal Free Hospital, London, UK

6. Institute of Liver Studies, King’s College Hospital, London, UK

7. Queen Elizabeth Hospital Birmingham, Birmingham, UK

8. Department of Surgery, University of Alberta, Edmonton, Canada

Key words: preservation, utilisation, ischaemia-reperfusion injury 


\section{Abbreviations:}

AST

ATP

DBD

DCD

DLI

EAD

HAT

$H \& E$

MAP

MELD

NMP

PAS

PNF

PRS

pSCS-NMP

RRT

SCS

UW
Aspartate aminotransferase

Adenosine triphosphate

Donor after brain stem death

Donor after circulatory death

Donor liver index

Early allograft dysfunction

Hepatic artery thrombosis

Haematoxylin \& eosin

Mean arterial pressure

Model for end-stage liver disease

Normothermic machine perfusion

Periodic acid Schiff

Primary non-function

Post reperfusion syndrome

Post-static cold storage normothermic machine perfusion

Renal replacement therapy

Static cold storage

University of Wisconsin

\section{Grants and Financial Support}

Carlo DL Ceresa was supported by a Medical Research Council (MRC) clinical research training fellowship. Consumables for the study were provided by OrganOx Ltd.

\section{Conflicts of Interest}

Carlo DL Ceresa has received income from OrganOx Ltd. for teaching and training and for carrying out normothermic organ preservations out of hours.

Simon Knight has previously received consultancy fees from Organox Ltd. for assisting with the design of clinical trials. 
Peter J. Friend and Constantin C. Coussios are full-time academics at the University of Oxford and also chief medical officer and chief technical officer, respectively, and shareholders in OrganOx Ltd, a spin-out company from the University of Oxford.

\section{Corresponding Authors}

Carlo DL Ceresa

Nuffield Department of Surgical Sciences

Oxford Transplant Centre

Churchill Hospital

Oxford

OX3 7LE

carlo.ceresa@nds.ox.ac.uk
Peter J Friend

Nuffield Department of Surgical Sciences

Oxford Transplant Centre

Churchill Hospital

Oxford

OX3 7LE

peter.friend@nds.ox.ac.uk 


\section{Abstract}

Introduction

Clinical adoption of normothermic machine perfusion (NMP) may be facilitated by simplifying logistics and reducing costs. This can be achieved by cold storage of livers for transportation to recipient centres before commencing NMP. The purpose of this study was to assess the safety and feasibility of post-static cold storage NMP (pSCS-NMP) in liver transplantation.

\section{Materials and Methods}

In this multi-centre prospective study, 31 livers were transplanted. The primary endpoint was 30-day graft survival. Secondary endpoints included: peak post-transplant aspartate aminotransferase (AST), early allograft dysfunction (EAD), post-reperfusion syndrome (PRS), adverse events, critical care and hospital stay, biliary complications and 12-month graft survival.

\section{$\underline{\text { Results }}$}

Thirty-day graft survival was 94\%. Livers were preserved for a total of $14 \mathrm{~h} 10 \mathrm{~min} \pm 4 \mathrm{~h} 46$ min; this included 6 h 1 min \pm 1 h 19 min of SCS before 8 h 24 min \pm 4 h 4 min of NMP. Median peak serum AST in the first 7 days postoperatively was 457 U/L (92 - 8669 U/L) and 4 livers (13\%) developed EAD. PRS was observed in 3 (10\%) livers. The median duration of initial critical care stay was $3(1-20)$ days and median hospital stay was $13(7-31)$ days. Seven patients (23\%) developed complications of grade IIIb severity or above and 2 (6\%) patients developed biliary complications; one bile leak and one anastomotic stricture with no cases of ischaemic cholangiopathy. Twelve-month overall graft survival (including death with a functioning graft) was $84 \%$.

\section{$\underline{\text { Conclusion }}$}


This study demonstrates that pSCS-NMP was feasible and safe which may facilitate clinical adoption. 


\section{Introduction}

Normothermic machine perfusion (NMP) is a novel preservation method which is rapidly generating clinical interest in the field of liver transplantation. NMP maintains the liver ex situ at $37^{\circ} \mathrm{C}$ in a functioning state through the delivery of oxygen and nutrition. This permits the objective assessment of organ function during preservation through lactate clearance, glucose metabolism, pH maintenance and bile production and quality (1). A primary objective of this technology is to minimise graft injury during preservation and to improve organ utilisation through the use of higher-risk organs without compromising outcomes. This is of paramount importance in the context of the current donor organ shortage, which leads to the death of up to $20 \%$ of patients on the waiting list before receiving a transplant $(2,3)$. The paucity of donor organs is exacerbated by the poor quality of many livers offered for transplantation. Organ donation increasingly involves older donors with co-morbidities and the global obesity epidemic results in more livers with significant steatosis. These high-risk, or "extended criteria”, livers have a lower tolerance of ischaemia, which is associated with a more severe reperfusion injury. The resilience of an extended criteria liver to recover from the physiological and metabolic traumas of brain death, organ procurement, cold preservation and implantation is diminished. Furthermore, along with primary non-function (PNF), extended criteria livers are at increased risk of acute and chronic rejection and biliary complications including ischaemic cholangiopathy, particularly in organs retrieved after circulatory determination of death (DCD) $(4,5)$.

Pilot studies have demonstrated the safety and feasibility of NMP in the clinical setting (6-8), followed by a multicentre phase III randomised controlled trial which demonstrated the superiority of NMP compared to standard static cold storage (SCS) in terms of biochemical markers of early graft injury and improved organ utilisation (9). In this, the only randomised trial of NMP so far reported, perfusion was implemented for the entire preservation period, 
from retrieval to transplantation, including transportation (continuous NMP). The logistics of continuous NMP, whilst achievable, can be challenging, particularly in the context of organs procured a long distance from the transplanting centre which may need to be transported by air. The clinical implementation of NMP may be facilitated by the use of NMP after a period of cold storage (10), allowing transport of organs in an ice-box, thereby simplifying logistics and reducing costs.

However, there is experimental evidence suggesting that rapid re-warming after a period of cooling can induce cellular injury $(11,12)$. Work in a porcine model comparing continuous NMP with a post-SCS approach (pSCS-NMP), demonstrated livers that were subjected to pSCS-NMP had increased markers of hepatocellular injury, sinusoidal endothelial cell dysfunction and Kupffer Cell injury (13). Conversely, two clinical studies investigating NMP of discarded or high-risk human livers have demonstrated evidence that pSCS-NMP can be implemented without any apparent detriment to the graft (when using physiological oxygen tensions) $(1,14,15)$ and the end-ischaemic approach has been used successfully in hypothermic machine perfusion studies $(16,17)$. However, it remains unclear whether the deleterious effects of cooling the graft can be mitigated by subsequent NMP, resulting in comparable outcomes to grafts preserved by continuous NMP.

The aim of this study was to formally assess the safety and feasibility of pSCS-NMP and to provide some insight into how this might compare to continuous NMP. 


\section{$\underline{\text { Materials and Methods }}$}

\section{Study Design and Ethical Approval}

In this prospective clinical study, livers were recruited by three UK transplant centres until 30 recipients were transplanted after the intervention. In order to test safety and feasibility, 30-day graft survival was selected as the primary outcome. The study was sponsored by the University of Oxford and approved by the London-Dulwich research ethics committee in accordance with the Helsinki Declaration (16/LO/2196) and registered prior to commencing recruitment (NCT03176433).

\section{Eligibility and Consent}

Whole livers from donors after brainstem death (DBD) and Maastricht category III DCD (18), aged at least 16 years were eligible. For DCD livers, functional warm ischaemia time (fWIT) commenced when either systolic blood pressure dropped below $50 \mathrm{mmHg}$ or oxygen saturation below 70\% until cold in situ perfusion. Recipients were suitable provided they were at least 18 years old and listed for an elective liver-only transplant. All recipients provided formal informed consent prior to recruitment. This included the recording the use of anonymized data for trial purposes and the collection of biological samples for further analysis. No patient identifiable data were collected.

The study protocol stipulated that livers should be cold-stored for 3 to 8 hours before being placed on the NMP circuit. These time limits were established in order to ensure the intervention was tested consistently and to reflect the UK average cold ischaemia times (3).

\section{pSCS-NMP Livers}

Livers were retrieved and transported in University of Wisconsin (UW) solution at $4^{\circ} \mathrm{C}$ according to standard practice. On arrival of the organ at the transplanting centre a standard liver back-table was performed (19). The coeliac artery, portal vein, inferior vena cava and bile 
duct were then cannulated and the liver flushed with 500ml Gelofusine (B Braun Ltd). The liver was then connected to the OrganOx metra ${ }^{\circledR}$ normothermic device as previously described (69).

The minimum NMP duration was set at 4 hours, the time taken for adenosine triphosphate (ATP) repletion in an animal DCD model (20) and to provide time for functional assessment. Livers could be perfused for a maximum of 24 hours, as per the regulatory approval of the device, governed by local operating room logistics. The duration of perfusion was at the discretion of the transplanting surgeon. Blood gas analysis, sampled from an outlet via the hepatic artery line, was performed after 15 minutes and one hour from the beginning of perfusion and then at least 4 hourly until the end of perfusion. Functional parameters, including: lactate clearance, glucose metabolism, $\mathrm{pH}$ maintenance, bile production, perfusate transaminase levels and flow rates, were available to the transplanting surgeon, as previously described $(1,14,15)$. The final decision to transplant the liver was made by the transplanting surgeon. Specific functional criteria were not pre-defined as optimal functional parameters are yet to be validated.

\section{Study End-Points}

The primary endpoint was 30-day graft survival, in order to test the safety and feasibility of the pSCS-NMP approach.

Secondary end-points included:

- Peak serum aspartate aminotransferase (AST) in the first 7-days post-transplant. This is a validated surrogate marker, predictive of PNF as well as graft and patient survival (21, 22). It is also associated with histological evidence of moderate to severe reperfusion injury $(23,24)$. 
- Early allograft dysfunction (EAD) (25): any one of: (i) bilirubin $>172 \mu \mathrm{mol} / \mathrm{L}$ on day 7 post-transplant; (ii) INR $>1.6$ on day 7 post-transplant; (iii) peak-AST $>2000 \mathrm{U} / \mathrm{L}$ during the first 7 days.

- Model for Early Allograft Dysfunction (MEAF) score (26): a validated continuous scoring model graded 0-10 comprising the highest serum alanine aminotransferase (ALT) and INR in the first 3 post-operative days and bilirubin value on day 3 following liver-transplantation. The MEAF score has been proposed as a superior predictor of transplant survival than EAD (27).

- PNF: irreversible graft dysfunction requiring emergency liver replacement during the first 10 days after liver transplantation, in the absence of technical or immunological causes.

- $\quad$ Post-reperfusion syndrome (PRS): a decrease in mean arterial pressure (MAP) of more than $30 \%$ from the baseline value for more than one minute during the first five minutes after reperfusion $(28,29)$.

- $\quad$ Need for renal replacement therapy (RRT).

- Duration of initial postoperative critical care and hospital stays.

- Adverse event rates and severity, graded according to the Clavien-Dindo classification (30).

- Biliary complications at 12-months: bile leak, anastomotic strictures and ischaemic cholangiopathy.

- Graft histology

- 12-month graft and patient survival

\section{Post-SCS NMP Liver Histology}

For the 30 transplanted pSCS-NMP livers, core biopsies were obtained at the end of SCS and at the end of NMP and fixed in formalin. Sequential biopsies in individual livers were taken 
from the same part of the liver. Sections were stained with haematoxylin and eosin (H\&E) and Periodic Acid Schiff (PAS) and scored by a consultant histopathologist, blinded to the time at which the biopsy was taken. H\&E sections were scored for small and large droplet macrovesicular steatosis (none/mild/moderate/severe), necrosis, fibrosis, sinusoidal leukocytosis and PAS sections scored for glycogen depletion (none/focal/scattered/confluent).

\section{Comparison with continuous NMP}

In order to contextualize the results from this study, we compared preservation parameters and early biochemical outcomes with livers preserved via continuous NMP. This comparator group comprises all of the NMP livers transplanted in the UK $(n=104)$ as part of a randomized trial comparing the efficacy of NMP compared to SCS (9). Livers in this study were transplanted at the same UK centres with identical inclusion and exclusion criteria. We included the whole cohort (rather than formal matching) to eliminate any risk of selection bias.

\section{Statistical Analysis}

The present study was primarily designed to investigate the safety and feasibility of the pSCSNMP approach to preservation. As such a formal sample size calculation to assess efficacy was not performed.

All analyses were performed using GraphPad Prism version 7 for Mac OS X (GraphPad Software, La Jolla California USA). A Student's t-test was used to analyse parametric, continuous variables and the Mann-Whitney $U$ test used for non-parametric continuous variables. Fisher's exact test was used for categorical variables. Pearson $r$ test was used to test for correlation. Continuous variables are presented as mean \pm standard deviation (SD) or median with range. A p value $<0.05$ is considered statistically significant. 


\section{$\underline{\text { Results }}$}

\section{Recruitment}

Between $4^{\text {th }}$ May and $18^{\text {th }}$ July 2017, 30 liver transplants were performed using donor organs subjected to pSCS-NMP during preservation. One further liver could not be perfused due to a leak from the NMP disposable circuit during priming resulting in the liver remaining cold stored. This liver is included in the results as part of an intention-to-treat analysis. In total, 51 livers were recruited to the study with 20 being excluded for the following reasons: nonproceeding DCD $(n=12)$; suboptimal appearance at retrieval $(n=4)$; poor function during $\operatorname{NMP}(n=3)$; change to a non-eligible super-urgent recipient $(n=1)$.

\section{Donor, Recipient and Preservation Characteristics}

The indications for transplantation were: primary sclerosing cholangitis $(n=6)$, non-alcoholic steatohepatitis $(n=6)$, hepatocellular carcinoma $(n=5)$, alcoholic liver disease $(n=5)$, hepatitis C cirrhosis $(n=4)$, autoimmune hepatitis $(n=2)$, primary biliary cirrhosis $(n=1)$, polycystic liver disease $(n=1)$ and Budd-Chiari $(n=1)$.

Twenty-three (74\%) DBD and 8 (26\%) DCD livers were transplanted from donors with a median age of $58(17-78)$. The median donor risk index (DRI) was $1.87(1.06-3.20)$ and median donor liver index (DLI), 1.33 (0.6 - 3.38). The median recipient age was 58 (25 - 73) with a median MELD score of $14(17$ - 24). For the DCD livers, the median fWIT was 16 min (12 - $28 \mathrm{~min})$ and the median UK-DCD risk score (31) was 7 (4 - 11).

The mean total preservation time of livers was 14 h 10 min \pm 4 h 46 min; this included 6 h 1 $\min \pm 1 \mathrm{~h} 19 \mathrm{~min}$ of SCS before $8 \mathrm{~h} 24 \mathrm{~min} \pm 4 \mathrm{~h} 4 \mathrm{~min}$ of NMP. The longest total preservation time was 25 h 39 min. During NMP, the mean hepatic arterial flow rate was $0.44 \pm 0.15 \mathrm{~L} / \mathrm{min}$ and portal venous flow rate was $1.08 \pm 0.12 \mathrm{~L} / \mathrm{min}$. After an initial bolus of sodium bicarbonate (10 - $40 \mathrm{ml}$ in the first $2 \mathrm{~h}$ of perfusion, according to $\mathrm{pH}$ ), perfusate $\mathrm{pH}$ increased from $6.99 \pm$ 
0.11 to $7.33 \pm 0.06(\mathrm{p}<0.0001)$. A significant reduction in perfusate lactate from $6.8 \pm 2.3$ $\mathrm{mmol} / \mathrm{L}$ to $0.9 \pm 0.5 \mathrm{mmol} / \mathrm{L}(\mathrm{p}<0.0001)$ and glucose from $24 \mathrm{mmol} / \mathrm{L}(13-39 \mathrm{mmol} / \mathrm{L})$ to $10 \mathrm{mmol} / \mathrm{L}(2-23 \mathrm{mmol} / \mathrm{L})(\mathrm{p}<0.0001)$ was observed over the course of NMP. Perfusate AST remained relatively stable from a median of $847 \mathrm{U} / \mathrm{L}(83 \mathrm{U} / \mathrm{L}-3379 \mathrm{U} / \mathrm{L})$ at the beginning of NMP to $1070 \mathrm{U} / \mathrm{L}(327 \mathrm{U} / \mathrm{L}-4122 \mathrm{U} / \mathrm{L})$ at the end of NMP ( $=0.29)$. There was no correlation between perfusate AST and peak serum AST $(r=0.04, p=0.89)$. Median bile production was $11 \mathrm{ml} / \mathrm{h}(0-50 \mathrm{ml} / \mathrm{h})$.

\section{Clinical Outcomes}

30-day Graft Survival (primary outcome)

Thirty-day graft survival was $94 \%$ in the study group. The two graft losses were due to acute hepatic artery thrombosis (HAT); both patients were successfully re-transplanted. The donor and preservation data for these livers are shown in Table 1.

There were no cases of PNF and the median duration of initial inpatient stay was 13 (7 - 31) days.

\section{Adverse Events}

Seven patients (23\%) developed complications of grade IIIb severity (Clavien-Dindo classification (30)) or above. These included surgical intervention (re-transplantation $(\mathrm{n}=2)$, bleeding $(n=1)$, corrective vascular intervention $(n=1)$ and sepsis control $(n=1))$ and RRT alone $(n=2)$. Eleven patients $(35 \%)$ developed more minor complications (2 (6\%) grade I, 7 (23\%) grade II and 2 (6\%) grade IIIa). Thirteen (42\%) patients had an uncomplicated postoperative course. 


\section{Early Biochemical Function}

The median peak serum AST in the first 7 days postoperatively was $457 \mathrm{U} / \mathrm{L}$ (92 - $8669 \mathrm{U} / \mathrm{L}$ ). There was no relationship between the duration of cold storage $(r=0.16, p=0.38)$ or total preservation time $(\mathrm{r}=0.1, \mathrm{p}=0.58)$ and peak serum AST.

Four of the 31 livers (13\%) developed EAD; 2 due to elevated peak AST only, one due to both elevated peak AST and elevated day 7 bilirubin and one due to elevated day 7 bilirubin only.

Recipient serum AST, bilirubin and INR in the first 6 months post-transplantation are shown in Figure 1A-C. The median MEAF score was 2.99 (0.74-8.85).

\section{Physiological Response to Preservation Injury}

Three patients (10\%) in the study experienced PRS immediately following the liver reperfusion through the portal vein. This was in the context of modest vasopressor use, with 21 patients (68\%) requiring a pre-reperfusion vasopressor infusion, 16 patients (52\%) requiring a postreperfusion vasopressor bolus and 19 patients (61\%) requiring a post-reperfusion vasopressor infusion. The median post-reperfusion lactate level was $3.95 \mathrm{mmol} / \mathrm{L}(2.6-8.3 \mathrm{mmol} / \mathrm{L})$. Five patients (16\%) required RRT in the first 30-days post-transplantation and the median duration of initial critical care stay was $3(1-20)$ days.

\section{Biliary Complications}

At 12-months follow-up, 2 patients experienced biliary complications; one with a biliary anastomotic leak and the other, an anastomotic stricture successfully managed by endoscopic retrograde cholangiopancreatography and stent insertion. No cases of ischaemic cholangiopathy were observed in the 31 patients. 


\section{2-month Patient and Graft Survival}

Twelve-month overall graft survival (including one death with a functioning graft) was $84 \%$ and patient survival was 90\%. Donor, preservation details and causes of all death-censored graft losses are shown in Table 1.

\section{Histology}

Histological assessment of the pSCS-NMP livers did not show any evidence of deterioration during NMP in terms of necrosis or fibrosis. Following the initial period of SCS, sinusoidal leukocytosis was observed in 50\% of livers. This fell to $17 \%$ of livers after NMP ( $p=0.03)$ (Figure 2A + B). In 56\% of livers, replenishment of glycogen stores was observed by at least one category (none, focal, scattered, confluent) after NMP (Figure 2C + D). Six livers displayed moderate/severe macrovesicular steatosis (predominantly large droplet) and the degree of steatosis did not appear to change during NMP.

\section{Livers Declined after NMP}

Three livers in the pSCS-NMP group were discarded following a period of NMP. The justifications for discard are shown in Table 2.

\section{Comparison with continuous NMP}

The pSCS-NMP study group and continuous NMP comparator group displayed similar donor and recipient characteristics (enrollment criteria and follow-up protocols were the same for the two studies) (Table 3). The addition of a period of cold storage prior to NMP in the pSCSNMP group significantly prolonged the total preservation time compared to continuous NMP (Table 4). Furthermore, some interesting differences were observed in the perfusion parameters between the groups with significantly higher hepatic arterial flow rates and significantly lower 
perfusate lactate levels at the beginning of preservation observed in the pSCS-NMP study group compared to continuous NMP comparator group (Table 4). Clinical outcomes appeared similar between the groups (Table 5). 


\section{$\underline{\text { Discussion }}$}

In this group of livers, we have demonstrated that pSCS-NMP is safe and feasible with $94 \%$ 30-day graft survival. The 6\% incidence of acute HAT observed in this study is in keeping with other published series' where an incidence of up to $9 \%$ is quoted (32-34). Neither case of hepatic artery thrombosis appeared to be related to the perfusion process and are discussed later.

Applying NMP after SCS has evident logistical and cost benefits. Removing the need to transport the device to and from a donor hospital reduces costs and avoids prolonging the donation procedure. In order to deploy NMP at the donor hospital, it is necessary to provide alternative transportation to support the extra equipment required and an extra member of the retrieval team may also be necessary. This will undoubtedly increase the cost associated with the organ retrieval process. Notably, Selzner et al also acknowledged that initiating NMP at the donor hospital, prolonged the procedure by 2 h (7) which may have implications on local operating lists. Although continuous NMP has been carried out in around 400 cases globally, transporting the liver on the device does add inherent risk; any problem on the device is likely to be much more difficult to resolve if it were to occur in transit. Furthermore, a multi-organ retrieval surgeon may not possess the necessary experience and expertise to perform a vascular reconstruction at the donor hospital prior to the commencement of NMP (7). The logistic benefits of NMP could be considerable; by facilitating prolonged preservation, centres would able to accept organs simultaneously or indeed plan elective operating more effectively. In the UK, from 2013 - 2016, a total of 163 livers were declined for logistical reasons alone (3). During this study, we observed a shift from out-of-hours operating with 22 (71\%) transplants being performed between 8am and 8pm; the start time was often delayed, ensuring the staff were well-rested. 
There is an ongoing pressure to increase utilization of higher-risk deceased donor organs; this is driven by increasing numbers of waiting list deaths $(2,35)$. There is an expanding body of evidence that NMP is of benefit, not only by reducing preservation associated reperfusion injury (9), but also allowing surgeons to assess the viability of an organ before transplantation $(1,14)$. It is not clear whether the benefit of NMP is restricted to higher-risk organs - certainly the livers in this study had a median DLI within the top quartile of UK donor livers (52\% of the transplanted pSCS-NMP livers), implying a 3-fold increase in the risk of graft loss compared to low-risk livers (36). It may not be possible to assess the benefit in low-risk organs until more experience has been gained in this context. Perhaps more importantly, livers in the upper quartile of donor risk (DLI) have a 34\% utilisation rate (36) and it is plausible that this technology, by removing the uncertainty surrounding these organs, may give surgeons the confidence to improve this rate of utilisation. This aligns with the observed increase in organ utilisation in the normothermic arm of the recently published randomised study of Nasralla et al (9).

Three livers were not transplanted in this study due to concerns over their ex situ function. In all of these cases, it was not one parameter in isolation, but a combination of factors which led to the decision to discard (Table 2). One liver was from a young DBD donor, and appeared to have mild steatosis on visual inspection. However, poor function during NMP (Table 2) prompted a biopsy of the graft which demonstrated $80 \%$ macrovesicular steatosis. This case illustrates the possible benefit of NMP in successfully discriminating between grafts and identifying those which are likely to have a poor post-transplant outcome (which may otherwise have been transplanted). In other cases, the decision to transplant the liver may not be as straight forward. For example, of four transplanted livers that failed to produce bile, two were ultimately lost (one HAT and one recurrent hepatitis C infection, rejection and cholestasis); 
these livers otherwise functioned well during NMP. Increasing evidence suggests that lack of bile production alone should not support discarding the liver $(1,9)$. Indeed, it is now becoming apparent that the quality of bile in terms of $\mathrm{pH}$, glucose and bicarbonate levels are more predictive of biliary complications than bile volume $(1,37)$.

The graft losses in this study do not appear to be attributable to the preservation method (Table 1). However, it is notable that the hepatic artery flow during NMP was low in one of the two livers which developed arterial thrombosis. Explant histology from this liver demonstrated marked medial muscular hypertrophy of the right and left hepatic arteries with narrow lumens with a normal common hepatic artery. In the case of sub-optimal hepatic artery flow, a doppler ultrasound scan during perfusion may be indicated to confirm vascular integrity and global organ perfusion. For the other liver that developed acute HAT, there were concerns about a small right hepatic artery upon implantation. The recipient was electively anticoagulated and the graft demonstrated good primary function. A spontaneous bleed from a diaphragmatic artery resulted in prolonged hypotension, thrombosis of the right hepatic artery and infarction of the right lobe of the liver.

This study has also identified some interesting comparisons between pSCS-NMP and continuous NMP. We observed a significantly higher arterial flow rate in the pSCS-NMP livers than in the continuous NMP comparator group during preservation $(0.44 \pm 0.15 \mathrm{~L} / \mathrm{min}$ vs. 0.29 $\pm 0.12 \mathrm{~L} / \mathrm{min}$, respectively; $\mathrm{p}<0.0001$ ). The reasons for this are unclear, although a previous study observed initial vasoconstriction after cold flush followed by vasodilatation during cold storage in a pig renal artery which appeared to be attributable to intracellular sources of calcium (38). If this phenomenon also occurs in the human hepatic artery, it may explain our observation, although this remains unproven. 
The perfusate lactate level after $15 \mathrm{~min}$ in the pSCS-NMP group $(7.3 \mathrm{mmol} / \mathrm{L}(1.9$ - 10.1 mmol/L)) was significantly lower than that of the continuous NMP group $(10.1 \mathrm{mmol} / \mathrm{L}(1.2$ $16.0 \mathrm{mmol} / \mathrm{L}))(\mathrm{p}<0.0001)$. It has previously been shown that livers do not reach $4^{\circ} \mathrm{C}$ until around 90 min of immersion in the ice box (39). It is possible that the continuous NMP livers have more active anaerobic metabolism during the back-table phase, as this takes place before complete cooling. Increased (anaerobic) metabolic activity may manifest as an increased lactate level at the beginning of perfusion. There is no evidence that this affects the liver's functional ability to clear the lactate or post-transplant outcome.

It also appears that early biochemical liver function between the groups is comparable although these results should be interpreted with caution as this study was not specifically designed or adequately powered to test this hypothesis.

In this study there is no evidence that prolonged NMP preservation is detrimental to the graft. There was no correlation observed between the duration of preservation and peak postoperative serum AST and histological analysis showed no new injury after NMP. There was a reduction in the clinical phenomenon of preservation injury after transplantation, as well as a reduction in sinusoidal leukocytosis after NMP, suggesting that NMP may reduce the inflammatory load within the liver. Recently, Jassem et al provided a mechanistic insight into how NMP may attenuate reperfusion injury by demonstrating an altered gene expression profile in NMP compared to cold-stored livers from pro-inflammation to pro-healing and regeneration (40). There may also be a benefit in a period of hypothermic machine perfusion prior to NMP, or “controlled re-warming” as was shown by Boteon et al where livers which had been cold stored for prolonged periods benefited from 2 hours of hypothermic oxygenated machine perfusion (HOPE) before NMP (12). Although these livers were not transplanted, HOPE + NMP livers 
achieved lower tissue expression markers of oxidative injury and inflammation as well as enhanced metabolic recovery compared to livers subjected directly to NMP (12).

This study is not without limitations. The number of recruited livers was small and the study is purely observational in nature. The study is not powered to detect any small differences that may be present between the pSCS-NMP and continuous NMP cohorts. To test equivalence to continuous NMP an appropriately powered randomised trial would be needed. The small sample size also precludes subgroup analyses, and it is possible that continuous NMP (as opposed to pSCS-NMP) may still be beneficial in the highest risk livers, in particular those with severe steatosis, where the pronounced adverse effects of cooling are well documented $(41,42)$. Use of a retrospective comparison with historical cohorts is a recognized limitation, although use of the same inclusion criteria and comparison with the entire UK contemporary cohort from the previous RCT does limit the risk of selection bias.

In summary, we have demonstrated that NMP after a period of SCS is both feasible and safe. This may have significant logistical and cost benefits that are likely to facilitate the uptake of NMP in routine clinical practice. 


\section{Acknowledgements}

The following organizations, groups and individuals made substantial contributions without which this trial could not have been completed successfully: NHS Blood and Transplant; the Clinical Trials and Research Governance Unit, University of Oxford; the Liver Transplant Coordinators, anaesthetists and liver unit physicians at the Royal Free Hospital, London Addenbrooke’s Hospital, Cambridge, and King’s College Hospital, London; D. Sharma, B. Davidson, M. Malago, R Ravikumar, R. Praseedom, P. Gibbs, A. Jah, N. Russell, S. Harper, J. Richards, L. Selves, Y. Puri, A. Zamalloa. Of course, thanks are also due to all of the donors' families and to the participating recipients. 


\section{Figure Legends}

Figure 1

Figure 1. Trend in recipient serum AST (A), bilirubin (B) and INR (C) in first 6-months posttransplantation. Data presented as median with IQR.

Figure 2

Figure 2. H\&E stained liver section demonstrating extensive sinusoidal leukocytosis after $6 \mathrm{~h}$ 42 min of SCS (as highlighted) (A) with resolution after 11 h 58 min NMP (B). PAS stained liver section demonstrating scattered glycogen depletion after 6 h 9 min of SCS (C) and complete replenishment of glycogen stores after 8 h 33 min of NMP (D). 


\section{References}

1. Watson CJE, Kosmoliaptsis V, Pley C, Randle L, Fear C, Crick K, et al. Observations on the ex situ perfusion of livers for transplantation. Am J Transplant. 2018.

2. Kim WR, Lake JR, Smith JM, Skeans MA, Schladt DP, Edwards EB, et al. OPTN/SRTR 2013 Annual Data Report: liver. Am J Transplant. 2015;15 Suppl 2:1-28.

3. NHSBT. Annual Report on Liver Transplantation. 2016.

4. Hoyer DP, Paul A, Gallinat A, Molmenti EP, Reinhardt R, Minor T, et al. Donor information based prediction of early allograft dysfunction and outcome in liver transplantation. Liver Int. 2015;35(1):156-63.

5. Foley DP, Fernandez LA, Leverson G, Anderson M, Mezrich J, Sollinger HW, et al. Biliary complications after liver transplantation from donation after cardiac death donors: an analysis of risk factors and long-term outcomes from a single center. Ann Surg. 2011;253(4):817-25.

6. Ravikumar R, Jassem W, Mergental H, Heaton N, Mirza D, Perera MT, et al. Liver Transplantation After Ex Vivo Normothermic Machine Preservation: A Phase 1 (First-inMan) Clinical Trial. Am J Transplant. 2016;16(6):1779-87.

7. Selzner M, Goldaracena N, Echeverri J, Kaths JM, Linares I, Selzner N, et al. Normothermic Ex Vivo Liver Perfusion Using Steen Solution as Perfusate for Human Liver Transplantation-First North American Results. Liver Transpl. 2016.

8. Bral M, Gala-Lopez B, Bigam D, Kneteman N, Malcolm A, Livingstone S, et al. Preliminary Single Centre Canadian Experience of Human Normothermic Ex Vivo Liver Perfusion: Results of a Clinical Trial. Am J Transplant. 2016.

9. Nasralla D, Coussios CC, Mergental H, Akhtar MZ, Butler AJ, Ceresa CDL, et al. A randomized trial of normothermic preservation in liver transplantation. Nature. 2018. 
10. Karangwa SA, Dutkowski P, Fontes P, Friend PJ, Guarrera JV, Markmann JF, et al. Machine Perfusion of Donor Livers for Transplantation: A Proposal for Standardized Nomenclature and Reporting Guidelines. Am J Transplant. 2016.

11. Bruinsma BG, Yeh H, Ozer S, Martins PN, Farmer A, Wu W, et al. Subnormothermic machine perfusion for ex vivo preservation and recovery of the human liver for transplantation. Am J Transplant. 2014;14(6):1400-9.

12. Boteon YL, Laing RW, Schlegel A, Wallace L, Smith A, Attard J, et al. Combined Hypothermic and Normothermic Machine Perfusion Improves Functional Recovery of Extended Criteria Donor Livers. Liver Transpl. 2018.

13. Reddy SP, Bhattacharjya S, Maniakin N, Greenwood J, Guerreiro D, Hughes D, et al. Preservation of porcine non-heart-beating donor livers by sequential cold storage and warm perfusion. Transplantation. 2004;77(9):1328-32.

14. Mergental H, Perera MT, Laing RW, Muiesan P, Isaac JR, Smith A, et al. Transplantation of Declined Liver Allografts Following Normothermic Ex-Situ Evaluation. Am J Transplant. 2016.

15. Watson CJE, Kosmoliaptsis V, Randle LV, Gimson AE, Brais R, Klinck JR, et al. Normothermic Perfusion in the Assessment and Preservation of Declined Livers Before Transplantation: Hyperoxia and Vasoplegia-Important Lessons From the First 12 Cases. Transplantation. 2017;101(5):1084-98.

16. Schlegel A, Muller X, Kalisvaart M, Muellhaupt B, Perera M, Isaac JR, et al. Outcomes of DCD liver transplantation using organs treated by hypothermic oxygenated perfusion before implantation. J Hepatol. 2018.

17. van Rijn R, Karimian N, Matton APM, Burlage LC, Westerkamp AC, van den Berg AP, et al. Dual hypothermic oxygenated machine perfusion in liver transplants donated after circulatory death. Br J Surg. 2017;104(7):907-17. 
18. Kootstra G, Daemen JH, Oomen AP. Categories of non-heart-beating donors. Transplant Proc. 1995;27(5):2893-4.

19. Makowka L, Stieber AC, Sher L, Kahn D, Mieles L, Bowman J, et al. Surgical technique of orthotopic liver transplantation. Gastroenterol Clin North Am. 1988;17(1):33-51. 20. Xu H, Berendsen T, Kim K, Soto-Gutierrez A, Bertheium F, Yarmush ML, et al. Excorporeal normothermic machine perfusion resuscitates pig DCD livers with extended warm ischemia. J Surg Res. 2012;173(2):e83-8.

21. Glanemann M, Langrehr JM, Stange BJ, Neumann U, Settmacher U, Steinmuller T, et al. Clinical implications of hepatic preservation injury after adult liver transplantation. Am J Transplant. 2003;3(8):1003-9.

22. Eisenbach C, Encke J, Merle U, Gotthardt D, Weiss KH, Schneider L, et al. An early increase in gamma glutamyltranspeptidase and low aspartate aminotransferase peak values are associated with superior outcomes after orthotopic liver transplantation. Transplant Proc. 2009;41(5):1727-30.

23. Karayalcin K, Mirza DF, Harrison RF, Da Silva RF, Hubscher SG, Mayer AD, et al. The role of dynamic and morphological studies in the assessment of potential liver donors. Transplantation. 1994;57(9):1323-7.

24. Gaffey MJ, Boyd JC, Traweek ST, Ali MA, Rezeig M, Caldwell SH, et al. Predictive value of intraoperative biopsies and liver function tests for preservation injury in orthotopic liver transplantation. Hepatology. 1997;25(1):184-9.

25. Olthoff KM, Kulik L, Samstein B, Kaminski M, Abecassis M, Emond J, et al. Validation of a current definition of early allograft dysfunction in liver transplant recipients and analysis of risk factors. Liver Transpl. 2010;16(8):943-9. 
26. Pareja E, Cortes M, Hervas D, Mir J, Valdivieso A, Castell JV, et al. A score model for the continuous grading of early allograft dysfunction severity. Liver Transpl. 2015;21(1):38-46.

27. Jochmans I, Fieuws S, Monbaliu D, Pirenne J. "Model for Early Allograft Function" Outperforms "Early Allograft Dysfunction" as a Predictor of Transplant Survival. Transplantation. 2017;101(8):e258-e64.

28. Hilmi I, Horton CN, Planinsic RM, Sakai T, Nicolau-Raducu R, Damian D, et al. The impact of postreperfusion syndrome on short-term patient and liver allograft outcome in patients undergoing orthotopic liver transplantation. Liver Transpl. 2008;14(4):504-8. 29. Chung IS, Kim HY, Shin YH, Ko JS, Gwak MS, Sim WS, et al. Incidence and predictors of post-reperfusion syndrome in living donor liver transplantation. Clin Transplant. 2012;26(4):539-43.

30. Dindo D, Demartines N, Clavien PA. Classification of surgical complications: a new proposal with evaluation in a cohort of 6336 patients and results of a survey. Ann Surg. 2004;240(2):205-13.

31. Schlegel A, Kalisvaart M, Scalera I, Laing RW, Mergental H, Mirza DF, et al. The UK DCD Risk Score: A new proposal to define futility in donation-after-circulatory-death liver transplantation. J Hepatol. 2018;68(3):456-64.

32. Mourad MM, Liossis C, Gunson BK, Mergental H, Isaac J, Muiesan P, et al. Etiology and management of hepatic artery thrombosis after adult liver transplantation. Liver Transpl. 2014;20(6):713-23.

33. Abou El-Ella K, Al Sebayel M, Ramirez C, Hussien R. Outcome and risk factors of hepatic artery thrombosis after orthotopic liver transplantation in adults. Transplant Proc. 2001;33(5):2712-3. 
34. Puliti Reigada CH, de Ataide EC, de Almeida Prado Mattosinho T, Boin I. Hepatic Artery Thrombosis After Liver Transplantation: Five-Year Experience at the State University of Campinas. Transplant Proc. 2017;49(4):867-70.

35. NHSBT. Organ Donation and Transplantation Activity Report 2015/16 2016

[Available from: http://www.odt.nhs.uk/pdf/activity-report/activity_report_2015_16.pdf.

36. Collett D, Friend PJ, Watson CJ. Factors Associated With Short- and Long-term Liver Graft Survival in the United Kingdom: Development of a UK Donor Liver Index. Transplantation. 2017;101(4):786-92.

37. Matton APM, de Vries Y, Burlage LC, van Rijn R, Fujiyoshi M, de Meijer VE, et al. Biliary Bicarbonate, $\mathrm{pH}$ and Glucose Are Suitable Biomarkers of Biliary Viability During Ex Situ Normothermic Machine Perfusion of Human Donor Livers. Transplantation. 2018.

38. Herrera B, Eisenberg G, Holberndt O, Desco MM, Rabano A, Garcia-Barreno P, et al. Paradoxical effects of temperature on vascular tone. Cryobiology. 2000;41(1):43-50.

39. Hertl M, Howard TK, Lowell JA, Shenoy S, Robert P, Harvey C, et al. Changes in liver core temperature during preservation and rewarming in human and porcine liver allografts. Liver Transpl Surg. 1996;2(2):111-7.

40. Jassem W, Xystrakis E, Ghnewa YG, Yuksel M, Pop O, Martinez-Llordella M, et al. Normothermic machine perfusion (NMP) inhibits proinflammatory responses in the liver and promotes regeneration. Hepatology. 2018.

41. Spitzer AL, Lao OB, Dick AA, Bakthavatsalam R, Halldorson JB, Yeh MM, et al. The biopsied donor liver: incorporating macrosteatosis into high-risk donor assessment. Liver Transpl. 2010;16(7):874-84.

42. Nativ NI, Maguire TJ, Yarmush G, Brasaemle DL, Henry SD, Guarrera JV, et al. Liver defatting: an alternative approach to enable steatotic liver transplantation. Am J Transplant. 2012;12(12):3176-83. 
